\title{
Marker zeigt, ob Chemotherapie nötig ist
}

Der Plasminogenaktivator vom Urokinasetyp (uPA) und dessen Inhibitor (Plasminogenaktivator-Inhibitor Typ 1, PAI-1) sind für das Eindringen von Tumorzellen in das Gewebe und die folgende Metastasierung von Bedeutung. Der Nutzen dieser Biomarker wurde prospektiv an fast 650 Patientinnen mit nodal-negativem Brustkrebs geprüft. Demnach haben Patientinnen mit niedrigen uPA/PAI-1-Werten (uPA $\leq 3 \mathrm{ng} / \mathrm{mg}$; PAI-1 $\leq 14 \mathrm{ng} / \mathrm{mg}$ ) eine sehr gute Prognose. Nach zehn Jahren lebten $87 \%$ der Frauen krankheitsfrei, die Gesamtüberlebensrate lag bei fast $90 \%$ ohne jegliche adjuvante systemische Therapie. Patientinnen mit hohen Werten (uPA > 3 ng/mg; PAI-1 > 14 ng/mg) hatten eine 10-Jahres-Rezidivrate von $23 \%$, Patientinnen mit niedrigen Werten eine Rezidivrate von nur $12,9 \%$. Patientinnen mit hohen Testwerten profitierten aber von einer adjuvanten Therapie mit Cyclophosphamid, Methotrexat plus 5-Fluorouracil (CMF). Die Rezidivrate ließ sich durch die CMF-Therapie im Vergleich zu „unter Beobachtung“ signifikant verringern $(32,1$ vs. 21,3\%; Hazard Ratio 0,48; $\mathrm{p}=0,019$ ).
Die Ergebnisse der Studie haben sich bereits in der „Interdiszisplinären Leitlinie für Diagnostik, Therapie und Nachsorge des Mammakarzinoms“ von 2012 niedergeschlagen. Danach kann bei niedriger uPA/PAI-1-Konzentration vor allem Patientinnen, die nach traditionellen Kriterien ein unklares Rezidivrisiko (G2) haben, eine adjuvante Chemotherapie erspart werden. Empfohlen wird die Bestimmung mittels ELISA im Gewebeauf- schluss aus frischem oder tiefgefrorenem Tumorgewebe. Autorin Nadja Harbeck erinnert daran, dass die Ergebnisse wohl nur für HER2-negative Patientinnen gelten, da HER2-positive Patientinnen inzwischen von der Behandlung mit Trastuzumab profitieren. In der Studie war der HER2-Status noch nicht erhoben worden.

Peter Leiner

Harbeck $\mathrm{N}$ et al. Ten-year analysis of the prospective multicentre Chemo-NO trial validates American Society of Clinical Oncology (ASCO)-recommended biomarkers UPA and PAI-1 for therapy decision making in node-negative breast cancer patients. Eur J Cancer. 2013 Mar 13. [Epub ahead of print]

\section{kurz notiert}

\section{Prostatakrebs-Gene bei Jüngeren}

Jeder 50. Betroffene ist bei der Diagnose eines Prostatakarzinoms noch keine 50 Jahre alt. Karzinome junger Patienten zeigen dabei andere und weniger Mutationen als Karzinome älterer Patienten [Weischenfeldt J et al. Cancer Cell. 2013;23(2):159-70]. Durch ein häufiges Rearrangement können testosteronabhängig regulierte Gene teilweise mit Krebsgenen fusionieren, die dann ihrerseits durch Testosteron aktivierbar sind. Deshalb fördern männliche Geschlechts- hormone die Krebsentstehung oder das Krebswachstum bei jüngeren Patienten, aber immer weniger bei Älteren: Ab 50 geht die Dichte dieser Rezeptoren zurück. Am Projekt „Früher Prostatakrebs" beteiligt sind die Martini-Klinik und das Universitätsklinikum Hamburg-Eppendorf, das EMBL, das DKFZ, das Nationale Centrum für Tumorerkrankungen (NCT) Heidelberg sowie das Max-Planck-Institut für Molekulare Genetik in Berlin.

\section{Pankreasoperation noch mit 85?}

15\% der Patienten mit Pankreaskarzinom sind 80 Jahre und älter. Soll man ihnen trotz erhöhtem Mortalitäts- und Komplikationsrisiko noch zu einer Pankreasoperation raten? Bei sorgfältiger Selektion der

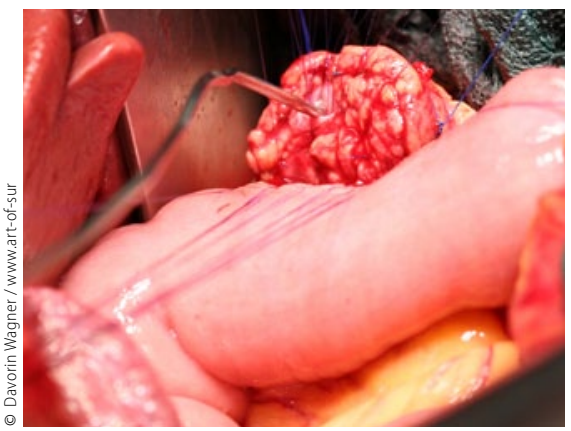

Pankreas vor der Reanastomosierung an eine Dünndarmschlinge nach Pankreaskopfresektion.
Patienten möglicherweise ja, wie eine retrospektive Untersuchung der Ruhr-Universität Bochum zeigt. Hier waren 1.705 Patienten mit einer Pankreaserkrankung operiert worden, 76 von ihnen waren 80 Jahre und älter. $80 \%$ der Patienten wurden wegen eines malignen Tumors operiert, $8 \%$ wegen eines benignen Tumors und $12 \%$ wegen einer chronischen Pankreatitis. 83\% der Patienten wurden in die ASA-Risikoklasse 3 eingruppiert. Bei allen Patienten bestand mindestens eine Komorbidität, am häufigsten eine kardiovaskuläre Erkrankung.

Postoperative Komplikationen ereigneten sich bei den Hochbetagten signifikant häufiger als in der Gesamtgruppe der Patienten mit Pankreasoperationen $(72,4$ vs. $42 \%$ ), besonders häufig handelte es sich um einen symptomatischen Pleuraerguss $(22,4 \%)$, einen Harnwegsinfekt (19,7\%), eine Arrhythmie (10,5\%) und eine Herzinsuffizienz (10,5\%). Chirurgische Komplikationen waren aber bei den betagten Patienten nicht häufiger, wohl aber eine verzögerte Magenentleerung.

$11,8 \%$ der operierten über 80 -Jährigen verstarben noch in der Klinik, 9,2\% innerhalb von 30 Tagen. Das mittlere Überleben lag bei 28,2 Monaten, bei den Krebspatienten bei 22,6 Monaten. Die häufigste Todesursache war die Krebserkrankung. $61,4 \%$ der Patienten überlebten ein Jahr postoperativ, 31,3\% zwei und 18,8\% drei Jahre. Interessanterweise waren alle verstorbenen Patienten Raucher oder schwere Ex-Raucher und hatten an COPD gelitten. Für die Autoren ein Hinweis darauf, dass für den Erfolg einer Pankreasoperation bei Hochbetagten vor allem eine sorgfältige Patientenselektion entscheidend ist.

Christine Starostzik

Belyaev O et al. Pancreatic Surgery in the Very Old: Face to Face With a Challenge of the Near Future. World J Surg. 2013;37(5):1013-20. 